\title{
Research on Dynamics Modeling Based on Human-structure Interaction
}

\author{
Zhifan Han \\ Department of Architectural Engineering, Guangdong Polytechnic College, Zhaoqing, 526000 \\ E-mail: hanzfhanzf@163.com
}

Keywords: human-structure dynamics; human dynamics model; resonance frequency; numerical simulation

\begin{abstract}
According to the human-structure interaction problem, an attempt is made to establish a new dynamic model of the human-structure coupling linkage system with multiple people. The crowd participated in the test and formed a simple supported beam joint system to study the dynamic characteristics of the system. The influence of the crowd on the dynamic characteristics of the simply supported beam. Studies have shown that people stand still on the platform structure, compared with the empty platform structure, and the resonance frequency of the human-structure system is reduced. The experimental numerical simulation results are consistent with the experimental results of foreign scholars. It is verified that the dynamic model of human-structure interaction is correct and of practical significance, which can fully verify the validity of the equivalent additional mass model before and after human body.
\end{abstract}

\section{Introduction}

With the rapid development of the civil engineering industry, the building structural materials become lighter, the span becomes larger, the height becomes higher, and the natural vibration frequency of the structure is reduced, so that the human-structure interaction becomes more obvious and the degree of damage increases. Report on the aspect.

Gao Shiqiao et al. [1] tested the structural acceleration changes when the single person stood in the beam span and stood still under the manual condition. The results show that when the person produces motion on the beam, the natural frequency of the structure is reduced, the damping is improved, and the human body is structurally fixed. The impact is even greater. Shaha et al. [2] performed modal tests based on the frequency response function and used discrete human-structure system models to simulate the interaction between pedestrians and structures. The test results show that for a pedestrian with a weight of $70 \mathrm{~kg}$, the natural vibration frequency of the human body is $2.75-3 \mathrm{~Hz}$, the modal damping ratio is $27.5-30 \%$. In order to solve the problem of high computational requirements of the MSD model, Ahmadi [3] derived the equivalent mobile load model for efficient calculation and described the analytical and numerical methods of the EMF system in detail and applied it to the modal analysis of simply supported beams. This method has certain requirements on the natural vibration frequency and the step frequency range of the human body, and the calculation is more complicated when applied to actual engineering. Nimmen et al. [4] assumed that the population 
would not be affected by structural motion and used the crowd model to study the influence of the vertical interaction of the human-structure on the vertical dynamic response of the structure. The study found that for the low-frequency structure, the damping ratio increased significantly. Large, the instantaneous frequency has been reduced.

In summary, the domestic research results in this area are relatively few, still in the preliminary research stage, and the existing literature at home and abroad is mostly the researcher-structure vertical system, and there are few explanations about the human-structural level system. In this paper, the human-structure vibration experiment platform is established for the horizontal vibration of human-structural system. The vibration characteristics of the platform structure, mass-structure system and human-structure system are compared and analyzed. The level of human (stationary standing)-structural system is preliminarily described. Vibration characteristics.

\section{Human-Structure-MTMD analysis model and MTMD parameter optimization design}

The low-order natural vibration frequency of the building will overlap with the pedestrian excitation frequency band [5], which leads to excessive vibration response of the building under human vibration. Therefore, it is necessary to verify the human vibration response of the building according to the design requirements of the building comfort. If the vibration response is too large (especially for structures with strict vibration comfort requirements such as office buildings, residential apartments, and high-precision instrument laboratories), it is necessary to design the vibration reduction.

The response of the structure under the excitation of the pedestrian is mainly controlled by the architectural mode with the modal frequency and the pedestrian excitation frequency band. For the building structure with the scattered natural frequency distribution, the resonance modes of the building can be simplified to a single degree of freedom. The system is controlled separately by a vibration damping system. When using MTMD to control building vibration, it is generally first identified by dynamic analysis of the building's self-vibration mode, which is coincident with or close to the pedestrian excitation band, and then the MTMD is used to control each mode.

\subsection{Experimental model and overview}

The Q345 steel with a height of $1.21 \mathrm{~m}$ is used; the top and bottom of the model steel plate has a length and width of $600 \mathrm{~mm} \times 600 \mathrm{~mm}$ and a thickness of $10 \mathrm{~mm}$; the model steel column adopts a hollow rectangular tube with a length and width of $50 \mathrm{~mm} \times 30 \mathrm{~mm}$ and a thickness of $2 \mathrm{~mm}$. It is 1 . $2 \mathrm{~m}$. The model steel beam is also a hollow rectangular tube with a length and width of $50 \mathrm{~mm} \times 30$ $\mathrm{mm}$ and a thickness of $2 \mathrm{~mm}$; the model support is anchored with screws. At the same time, the horizontal $x$ direction is defined as the short side of the cross section of the column tube, the horizontal y direction is the long side direction, and the vertical $\mathrm{z}$ direction is the straight side direction.

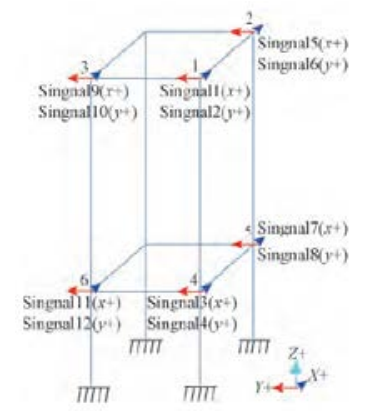

Fig.1 Human-Building-MTMD System Vibration Model 
The data acquisition and analysis system used in the experiment is the Danish B \& KpulseTM multi-channel analysis system 3560D, in addition to 12 4381-v accelerometers, 32692 amplifiers and 3 3050-A-040 modules. Wait. The arrangement of the vibrators is shown in Figure 1 (named after signal 1 - 12), where the horizontal $x$ direction is represented by an odd number and the horizontal y direction is represented by an even number.

\subsection{Human-structure interaction equation of motion}

Assume that the pedestrian building plane span $\mathrm{L}$, the elastic modulus $\mathrm{E}$, the moment of inertia is $\mathrm{I}, \mathrm{m}$ is defined as the mass per unit length of the simply supported beam, the body mass of the human body is mp, the body stiffness $\mathrm{kp}$, and the human body damping cp. The beam deflection $\mathrm{y}$ $(\mathrm{x}, \mathrm{t})$ is calculated from the static equilibrium position. The speed of the human body moving along the beam is $v$, and the displacement function of the human body is yp $(x, t)$. The human body keeps in contact with the simply supported beam, and the excitation on the beam is completely caused by the walking of the human body [6]. For a general simply supported beam, the bending vibration equation can be expressed as:

$$
\frac{\partial}{\partial x^{2}}\left[E I(x) \frac{\partial^{2} y(x, t)}{\partial x^{2}}\right]+\rho S(x) \frac{\partial^{2} y(x, t)}{\partial t^{2}}+C(x) \frac{\partial y(x, t)}{\partial t}=f(x, t)
$$

Using the orthogonality of the modal function, the mode decomposition method is simplified:

$$
y(x, t)=\sum_{n=1}^{N} q_{n}(t) \phi_{n}(x)
$$

Where $q_{n}$ is the nth-order generalized coordinate and $\phi_{n}$ is the nth-order modal function of the beam? In this paper, only the Euler simply supported beam is analyzed, and generalized mass, generalized stiffness and generalized modal force are introduced. Assuming that the simply supported beam, structure damping is proportional damping, equation (1) can be simplified as [6]:

$$
\ddot{q}_{n}(t)+2 \zeta_{n} \omega_{n} \dot{q}_{n}(t)+\omega_{n}^{2} q_{n}(t)=F_{n}(t)
$$

$M_{n}=\int_{0}^{L} \rho S(x)\left[\phi_{n}(x)\right]^{2} d x$ is the n-stage generalized mass of the structure, and $\zeta_{n}=c / 2 m \omega_{n}$ is the nth-order damping ratio.

$\omega_{n}$ is the nth order circular frequency, and the generalized modal force is expressed as $F_{n}(t)=\int_{0}^{L} \frac{f(t)}{M_{n}} \phi_{n}(x) d x$.

The modal function is regularized, the generalized mass of the structure is all normalized, the damping and stiffness matrices are all diagonalized at the same time, and $\phi_{n}(x)=\phi_{n}(x) / \sqrt{M_{n}}$, then equation (3) becomes:

$$
\ddot{q}_{n}(t)+2 \zeta_{n} \omega_{n} \dot{q}_{n}(t)+\omega_{n}^{2} q_{n}(t)=\int_{0}^{L} f(t) \phi_{n}(x) d x
$$

\section{Influence of limited individuals on dynamic characteristics of simply supported beam structures}

In general, although the civil structure is resident, its modal characteristics are still dominated by 
low-order modes. Firstly, the first-order modal characteristics of the beam of the human-beam joint system are simulated and compared with the first-order modal characteristics of the empty simply supported beam. The influence of the human on the dynamic characteristics of the simply supported beam can be estimated.

\subsection{Dynamic characteristics of a single individual- simply supported beam}

Let the single person position $\mathrm{x} 1$ change from 0 to $\mathrm{L}$. Using the new dynamic model for the simulation calculation, Figure 2 shows the relationship between the first-order frequency and the modal damping ratio of the beam of the single human-beam joint system and the human position $\mathrm{x}_{1}$.

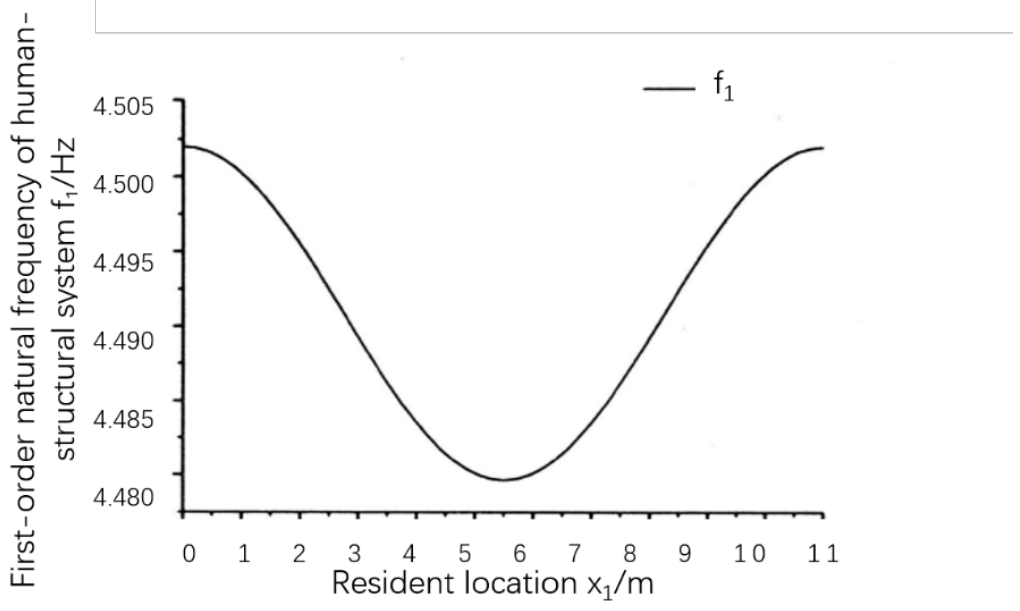

Fig. 2 The first-order frequency diagram of the beam of the single-girder joint system

The first-order frequency and damping ratio of the joint system beam are closely related to the human position $\mathrm{x} 1$. When the person is near the end of the beam, the first-order frequency value is slightly larger. As the person approaches the beam midpoint, it gradually decreases, and when the person reaches the beam midpoint, it is the minimum value. The modal damping ratio changes in the opposite direction. This phenomenon is explained as: when a person is located near the end of the empty beam, the influence of the human on the first-order modal characteristic of the beam is small, but the local additional constraint effect is generated. The first-order modal stiffness of the beam of the joint system increases with the frequency increase. When the person is located near the midpoint of the beam, it means that the person is located on the peak of the first-order mode of the beam, which has a significant influence on the mode characteristics of the order, and increases the peak and damping of the mode, which is represented by the beam of the joint system. The stiffness of the mode is reduced, and the frequency is reduced and the damping ratio is significantly increased. When two different positions of a single person are symmetric about the midpoint of the beam, then any one of the two locations at the two locations has exactly the same effect on the dynamic characteristics of the beam.

\subsection{Influence of limited individuals on modal characteristics of simply supported beams}

The simulation calculations show that if the mm individuals are crowded together without gaps, the influence of the crowd on the dynamic characteristics of the beam is similar to that of a single person; on the other hand, if the mm person is evenly spread on the simply supported beam, the crowd the effect is similar to the effect of distributing loads on the beam. In fact, the population of $\mathrm{mm}$ is different from the individual and different from the distributed load. To this end, an important parameter is newly defined: the population gap parameter $\varepsilon$-the center line of each human body in 
the crowd is separated by the gap $\varepsilon$. According to the human biodynamics study, the value of the gap $\varepsilon$ is roughly 1 to 2 . The normal human body width is reasonable, and $\varepsilon$ can be taken. $=0.4$ to $0.8 \mathrm{~m}$. Imagine the most dangerous conditions. The crowd is distributed near the mid-span with the greatest deflection of the simply supported beam, and the distribution of the human bodies is symmetric about the mid-span. Using the new dynamic model for simulation calculation, analyzing the dynamic characteristics of different numbers of human-beam joint systems, and deeply studying the influence of $\varepsilon$ and the number of people $\mathrm{mm}$ on the first-order modal characteristics of the beam, Figure 3 shows 2, 3, 4, 5 people - The curve of the first-order modal behavior of the beam of the simply supported beam joint system with $\varepsilon$.

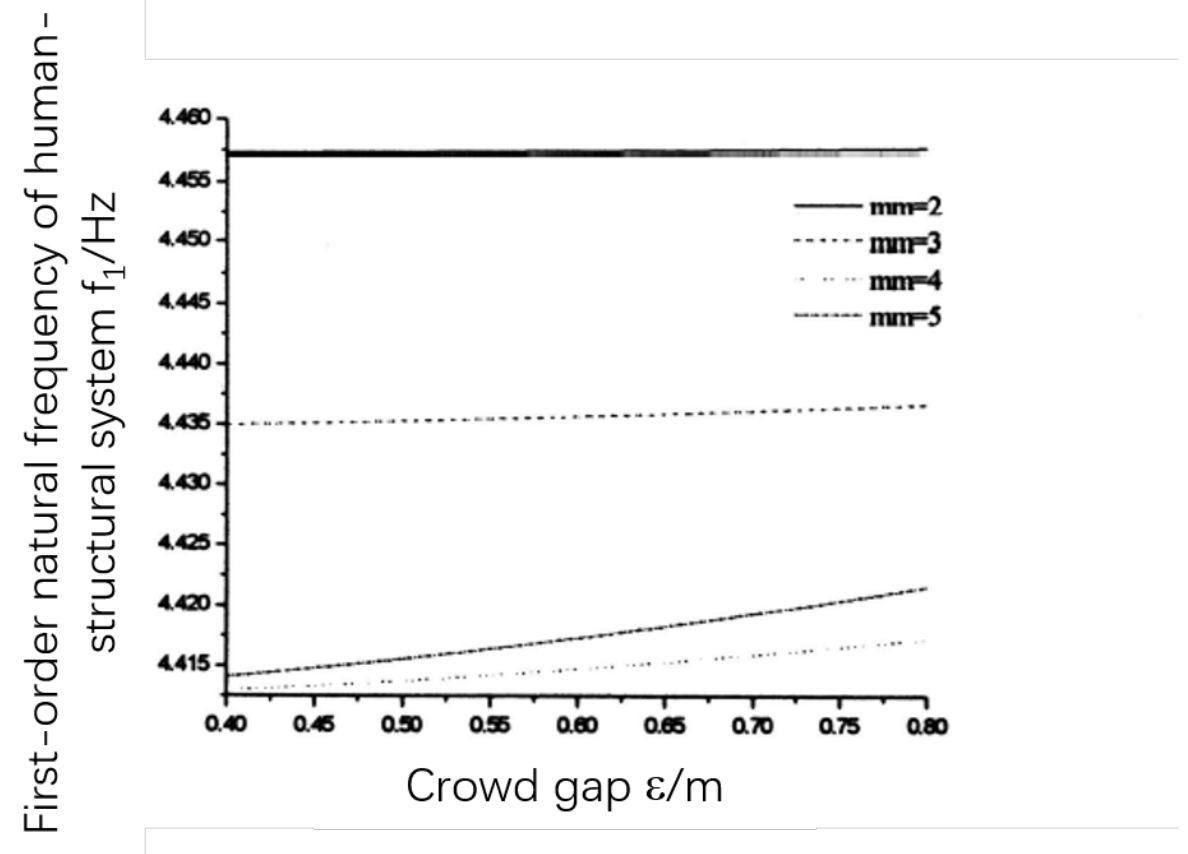

Fig.3 The relationship between the first-order modal characteristics and $\varepsilon$ of beams of different populations

\section{MTMD damping effect research}

The human body is simplified to the MMSD model and the MSD model. The curve of the first two orders of instantaneous damping ratio of the pedestrian when passing through the simply supported beam is shown in Fig. 3. Since the trends in the two types of simplified models are consistent, the peaks are displayed only in the figure. The value in parentheses is the calculated value under the MMSD model. The maximum first-order damping ratio of the structure under MMSD and MSD model is $0.45 \%$, and the maximum value of second-order damping ratio is $0.41 \%$. The vertical interaction effect of human-structure is obvious. As the pedestrian frequency increases, the damping ratio increases gradually. The instantaneous damping ratio changes in the two simplified models are only $2.86 \%$. It can be seen that the difference in structural damping ratio variation between the two types of simplified models is small. The MMSD model is consistent with the variation under the MSD model, and only the peaks are displayed simultaneously in the graph. The value in parentheses is the calculated value under the MMSD model. The next-order minimum instantaneous frequency of the MMSD model is $1.9928 \mathrm{~Hz}$, the second-order minimum instantaneous frequency is $7.99918 \mathrm{~Hz}$, the next-order minimum instantaneous frequency of the MSD model is $1.9928 \mathrm{~Hz}$, and the second-order minimum instantaneous frequency is $7.9971 \mathrm{~Hz}$. The instantaneous frequency variation under two simplified models the difference in value is only 
$0.03 \%$ at most, and the difference in instantaneous frequency variation of the structure under the two simplified models is small. Don't be small, in line with the results of the previous discussion.

\section{Conclusion}

In view of the increasingly significant human-structural system problem, this paper establishes a human-structure vibration experimental platform. By studying the horizontal vibration characteristics of the human-structural system (human stationary standing), the following conclusions are summarized: (1) Contrast mass-structure system and platform the horizontal natural frequency of the structure indicates that the horizontal self-vibration frequency of the mass-structure system of the additional mass is significantly reduced. 2) Comparing the platform structure with the human-structure system, it shows that the horizontal natural vibration frequency of the human-structure system is only slightly reduced, and the effect is not obvious. (3) Comparing the mass-structure system and the human-structure system, it shows that there are significant differences between the dynamic characteristics of the two. When considering the dynamic characteristics of the structure, only simplify it to the mass block attached to the structure is not correct.

\section{Acknowledgements}

The project is supported by the Science and Technology Project of Guangdong Polytechnic College (GKJ2018002).

\section{References}

[1] Ding Jinxue, Jin Fengjun, Wang Wei, et al. Competitive game between high-speed rail and civil aviation and their spatial effects: Take Beijing-Shanghai high-speed rail as an example. Economic Geography, Vol. 5 (2013) No. 33, p. 104-110.

[2] Gao Shiqiao, Wang Dong, Niu Shaohua. Dynamic Analysis of Human-Structure Coupling System. Journal of Beijing Institute of Technology, Vol. 3 (2013) No. 33, p. 105-110.

[3] Shaha AR, Silver CE, Angelos P, et al. The central compartment - Center of controversy, confusion, and concern in management of differentiated thyroid cancer. European Journal of Surgical Oncology the Journal of the European Society of Surgical Oncology \& the British Association of Surgical Oncology, Vol. 11 (2017) No. 43, p. 29-33.

[4] Ahmadi E, Caprani C C, Heidarpour A. An equivalent moving force model for consideration of human structure interaction. Applied Mathematical Modelling, Vol. 12(2017) No. 26, p. 526-545.

[5] VAN Nimmen K, Lombaert $G$, DeRoeck G, Van den Broeck P. The impact of vertical human-structure interaction on the response of footbridges to pedestrian excitation. Journal of Sound and Vibration, Vol. 2(2017) No. 40, p. $104-121$.

[6] Sachse R. Modeling effects of human occupants on modal properties of slender structures. The Structural Engineer, Vol. 10(2002) No. 35, p. 21-23.

[7] [Chen Zhou. Research on crowd-bridge coupled vibration and parameter analysis [J]. Journal of South China University of Technology: Natural Science Edition, Vol. 5(2014) No. 42, p. 75-82. 